\title{
Predict-prevent control method for perturbed excitable systems
}

\author{
Marzena Ciszak, ${ }^{1}$ Claudio R. Mirasso, ${ }^{2}$ Raúl Toral, ${ }^{2}$ and Oscar Calvo ${ }^{3}$ \\ ${ }^{1}$ C.N.R.-Istituto Nazionale di Ottica Applicata, L.go E. Fermi 6, 50125 Florence, Italy \\ ${ }^{2}$ Instituto de Física Interdisciplinar y Sistemas Complejos (IFISC), CSIC-UIB, Campus UIB, E-07122 Palma de Mallorca, Spain \\ ${ }^{3}$ Departament de Física, Universitat de les Illes Balears, E-07122 Palma de Mallorca, Spain \\ (Received 15 July 2008; revised manuscript received 3 February 2009; published 3 April 2009)
}

\begin{abstract}
We present a control method based on two steps: prediction and prevention. For prediction we use the anticipated synchronization scheme, considering unidirectional coupling between excitable systems in a master-slave configuration. The master is the perturbed system to be controlled, meanwhile the slave is an auxiliary system which is used to predict the master's behavior. The prevention is obtained by sending a control signal to the master system, which temporarily lowers its excitability threshold and prevents its future reaction to the external perturbation. We demonstrate theoretically and experimentally that an efficient control may be achieved.
\end{abstract}

DOI: 10.1103/PhysRevE.79.046203

PACS number(s): 05.45.Gg, 05.40.Ca, 45.80.+r, 87.19.1r

\section{INTRODUCTION}

A classical problem in engineering science is the control of dynamical systems [1]. While earliest applications in this field aimed to inhibit the instabilities in electronic devices, control studies turned, later on, into other areas. Of particular interest are the applications to medicine since transitions from regular to irregular oscillations of many organs of the human body have been found to be associated with a diseased behavior. As an example, the heart beat has been found to undergo chaotic behavior during arrhythmias and other heart diseases [2].

One of the best known methods for stabilizing unstable periodic orbits is the one introduced by Ott $e t$ al. [3]. It uses a small perturbation in some parameter of the system to stabilize an unstable orbit into a periodic one. The delayedfeedback control, introduced by Pyragas [4], intends the same but uses a self-feedback loop and works when the feedback delay time is close to the period of the unstable orbit that one aims to stabilize. It has been experimentally implemented in optical systems [5], chaotic flows [6], and cardiac systems [7] among others. Control of chaotic dynamics with external nondelayed-feedback stimuli has been reported in many models including those for neural systems [8] and heart muscle [9].

In this paper we present a control method with particular application, but not necessarily limited, to excitable systems. These systems have been used to model the behavior of many cell types, including the typical spiking dynamics of heart cells and neurons. Excitable systems are characterized by a highly nonlinear response to an external perturbation: if the perturbation is sufficiently small, the system returns smoothly to its steady state; if the perturbation exceeds a certain threshold, the system fires an excitable spike, sometimes called the action potential. During a refractory period following the spike, perturbations of moderate amplitude do not alter much the dynamics of the system. According to the classification of neural dynamics introduced by Hodgkin, class 1 excitable systems undergo a saddle-node bifurcation whereas class 2 excitable systems undergo a Hopf bifurcation. Although excitable systems can involve a large number of variables (as in the case of neurons), their essential features can be captured with a much more reduced description. We will be using in this paper the one-variable Adler (phase) and the two-variable FitzHugh-Nagumo systems as examples. They are very simple models of class 1 and class 2 excitable dynamics, respectively. The proposed control method relies on the phenomenon of anticipated synchronization. Synchronization refers to the collective timing of coupled systems, from simple oscillators and clocks to some vital functions found in living organisms, among many others $[10,11]$. Anticipated synchronization refers to a particular regime, first studied by Voss [12], which appears in unidirectionally coupled systems in a master-slave configuration. In this regime, two dynamical systems synchronize in such a way that the slave system, $\mathbf{y}(t)$, anticipates the trajectory of the master, $\mathbf{x}(t)$. Taking advantage of anticipated synchronization we propose a control method designed to correct unwanted dynamical behaviors. We apply the method to externally perturbed excitable systems. First, we show how it can be implemented to suppress unwanted spikes produced by the effect of external random perturbations in the prototype Adler system. We then prove that the control method can also work under nonideal conditions, such as the presence of noise in the dynamics and parameter mismatch between master and slave. Finally, we demonstrate experimentally the efficiency of the proposed method by experiments in electronic circuits whose dynamics mimics that of the FitzHughNagumo set of equations.

\section{MODEL}

Our control scheme uses the anticipated synchronization scheme, in which master, $\mathbf{x}(t)$, and slave, $\mathbf{y}(t)$, systems follow the following general evolution equations:

$$
\begin{gathered}
\dot{\mathbf{x}}(t)=\mathbf{f}[\mathbf{x}(t)]+I(t)+\epsilon(t), \\
\dot{\mathbf{y}}(t)=\mathbf{f}[\mathbf{y}(t)]+\mathbf{K}[\mathbf{x}(t)-\mathbf{y}(t-\tau)] .
\end{gathered}
$$

The function $\mathbf{f}[\mathbf{x}]$ defines the dynamical system under consideration, $\mathbf{K}$ is a general coupling function satisfying 


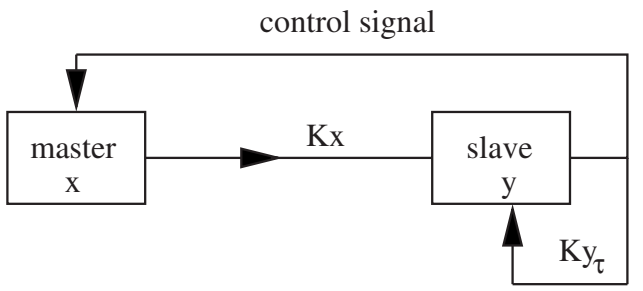

FIG. 1. Schematic presentation of the predict-prevent control method. The input signal $I(t)$ is applied only to the master system $\mathbf{x}$ while its influence on the slave $\mathbf{y}$ is indirectly through the unidirectional coupling Kx. The slave dynamics has a delayed loop $\mathbf{K} \mathbf{y}_{\tau} \equiv \mathbf{K y}(\mathbf{t}-\tau)$. The control signal is applied whenever a condition for the slave variable is satisfied.

$\mathbf{K}[\mathbf{0}]=\mathbf{0}$, and $\tau$ is the delay time in the feedback loop of the slave. The function $I(t)$ represents an external perturbation accounting for unexpected inputs acting upon the master dynamics. $\boldsymbol{\epsilon}(t)$ is the control function added, when required, to the master in order to suppress unwanted behavior, as we will explain in detail later. As proven by Voss [12], the manifold $\mathbf{y}(t)=\mathbf{x}(t+\tau)$, i.e., the slave anticipates by a time $\tau$ the dynamics of the master, can be a (structurally) stable solution of Eqs. (1) and (2) in the unperturbed case $I=\epsilon=0$. Anticipated synchronization has been studied theoretically and experimentally in many systems [13-22] (see also [23] for a short review). In this paper, we use the aforewritten version of the dynamical equations in order to suppress spiking in the master system induced by a random perturbation $I(t)$. The main idea is that the decision to activate the control procedure $\epsilon(t)$ is triggered by the behavior of the slave system. Since the slave system anticipates the behavior of the master, the control procedure can act earlier and then can be more effective than the case where its activation is triggered by the dynamics of the master.

\section{NUMERICAL RESULTS}

\section{A. Suppression of a single spike}

For the sake of concreteness, we start by considering two unidirectionally coupled Adler systems (phase equations)

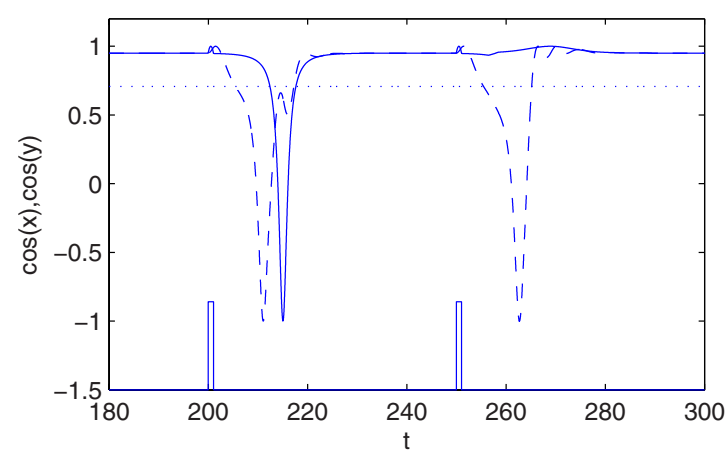

FIG. 2. (Color online) Evolution of the master system $\cos [x(t)]$ (solid line) and the slave system $\cos [y(t)]$ (dashed line) obtained from a numerical integration of Eqs. (3) and (4). External perturbations of intensity $I_{0}=0.64$ are applied at times $t=200$ and $t=250$ (shown at the bottom of the figure). In the first perturbation ( $t$ $=200$ ) we see clearly that the slave anticipates the dynamics of the master. In the second perturbation $(t=250)$ the spike of the master has been suppressed by applying a correcting pulse of amplitude $\epsilon_{0}=0.04$ when the trajectory of the slave crosses the reference level $\cos \left(x_{0}\right)=0.707$ (dashed horizontal line). We have used the parameters $\mu=0.95, K=0.6, \tau=1.5$, and a reaction time $t_{R}=1$.

[24] in the general master-slave configuration introduced before,

$$
\begin{gathered}
\dot{x}(t)=\mu-\cos [x(t)]+I(t)+\epsilon(t), \\
\dot{y}(t)=\mu-\cos [y(t)]+K \sin [x(t)-y(t-\tau)] .
\end{gathered}
$$

$\mu, K$, and $\tau$ are constant parameters. Throughout the paper, we will take the values $\mu=0.95, K=0.6$, and $\tau=1.5$, which satisfy the conditions for anticipated synchronization $[17,23]$. Note that the external perturbation $I(t)$ is applied only to the master system $x(t)$ while it acts on the slave system $y(t)$ only indirectly through the coupling term. We use a $2 \pi$ periodic function for coupling, appropriate for the angular-type variables we consider. The slave is driven by the master and subject to its own feedback loop, of strength $K$, producing the anticipated synchronization regime. As dis-
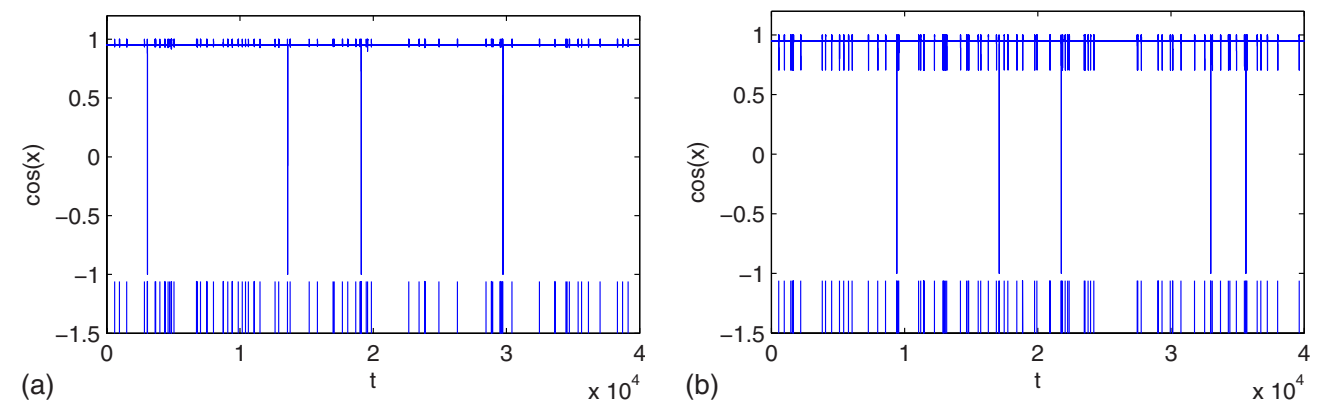

FIG. 3. (Color online) Correction of a series of spikes. The external perturbation $I(t)$ consists of a series of random pulses (vertical lines al the bottom of both panels) following a Poisson distribution with a given mean value $\langle t\rangle=500$. Independent noises of intensity $D=0.01$ have been added both to the master and to the slave. Left panel: control of magnitude $\epsilon_{0}=0.3$ with a response time $t_{R}=1$ using a criterion based on the slave variable $y$, as explained in the text. There are 72 excitations altogether and the method manages to suppress the spikes in all but four cases. Right panel: control of magnitude $\epsilon_{0}=0.85$ with the response time $t_{R}=0$ and a control based on the master variable $x(t)$. In this case there are 85 excitations, and the control method allows suppressing all but five spikes. In both cases we take $t_{\text {rec }}=5$ and other parameters as in Fig. 2. 

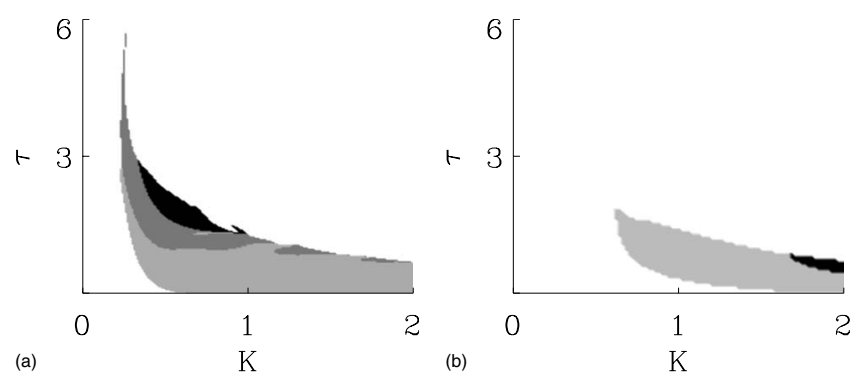

FIG. 4. Intensities of anticipation times for unidirectionally coupled master and slave with $\mu=0.95$ in the parameter space $(\tau, K)$. White region corresponds to the unstable or retarded slave (no control possible), other colors corresponds to the anticipation times $0<\tau<1$ (light gray), $1<\tau<6$ (dark gray), and $\tau>6$ (black). The perturbation magnitudes are $I_{0}=1.635$ (left panel) and $I_{0}=2.8$ (right panel).

cussed in [19], the feedback term lowers the excitability threshold of the slave and allows it to react to the perturbation faster than the master. Hence, the slave is used to anticipate the future response of the master to this perturbation. When an unwanted spike appears in the dynamics of the slave before it does in the master, a control mechanism is triggered and an appropriate control stimulus $\epsilon(t)$ is sent to the master in order to prevent that event. The whole scheme is shown in Fig. 1. We now give some specific details of the method.

It is easy to see that the master system $x(t)$ is excitable for $|\mu|<1$, with fixed points at $x_{ \pm}= \pm \arccos (\mu)$, and oscillatory for $|\mu|>1$. If the system is at the stable point $x(t)=x_{-}$and a perturbation acts upon it for a short time, the way in which variable $x(t)$ returns to the stable value depends on the magnitude of the perturbation. Let us assume that we apply a single, deltalike, perturbation of magnitude $I_{0}$ at time $t_{0}>0$, i.e., we take $I(t)=I_{0} \delta\left(t-t_{0}\right)$, when the system is at the stable fixed point $x\left(t_{0}\right)=x_{-}$. The effect of such delta function perturbation is to instantly change at $t_{0}$ the value of $x(t)$ such that $x\left(t_{0}^{+}\right)=x\left(t_{0}^{-}\right)+I_{0}$. The subsequent dynamics depends on the magnitude of $I_{0}$. If $I_{0}<2 \arccos (\mu)$ the system returns smoothly to the fixed point $x_{-}$, whereas if $I_{0}>2 \arccos (\mu)$ the variable $x(t)$ executes a full rotation (a spike) and returns to the equivalent point $2 \pi+x_{-}$. It is the aim of our control method to prevent those spikes. We do this by adding to the dynamics of the master [Eq. (3)] a small corrective signal $\epsilon(t)=-\epsilon_{0} \delta\left(t-T_{0}\right)$ of magnitude $\epsilon_{0}$ at time $T_{0}$. The time $T_{0}$ is determined by two factors: a correction criterion and a response time. The correction criterion determines whether the corrective signal is sent, and the response time $t_{R}$ is the time it takes the correction action to act after it has been required. We also consider that the control mechanism has a recovery time $t_{\text {rec }}$ such that $t_{\text {rec }}$ must elapse after a control signal has been applied before another control signal can be activated.

Let us first consider a simple control method in whic $\mathrm{h}$ the correction criterion is that the variable $x(t)$ crosses a threshold value $x_{0}$. If this happens at time $t_{1}$, then at time $T_{0}=t_{1}+t_{R}$ the correction acts such that $x\left(T_{0}^{+}\right)=x\left(T_{0}^{-}\right)-\epsilon_{0}$, and it will be considered effective if $x(t)$ is brought back to the stable region. The sooner the correction action is taken, the smaller the magnitude of the correction $\epsilon_{0}$ is needed. In any

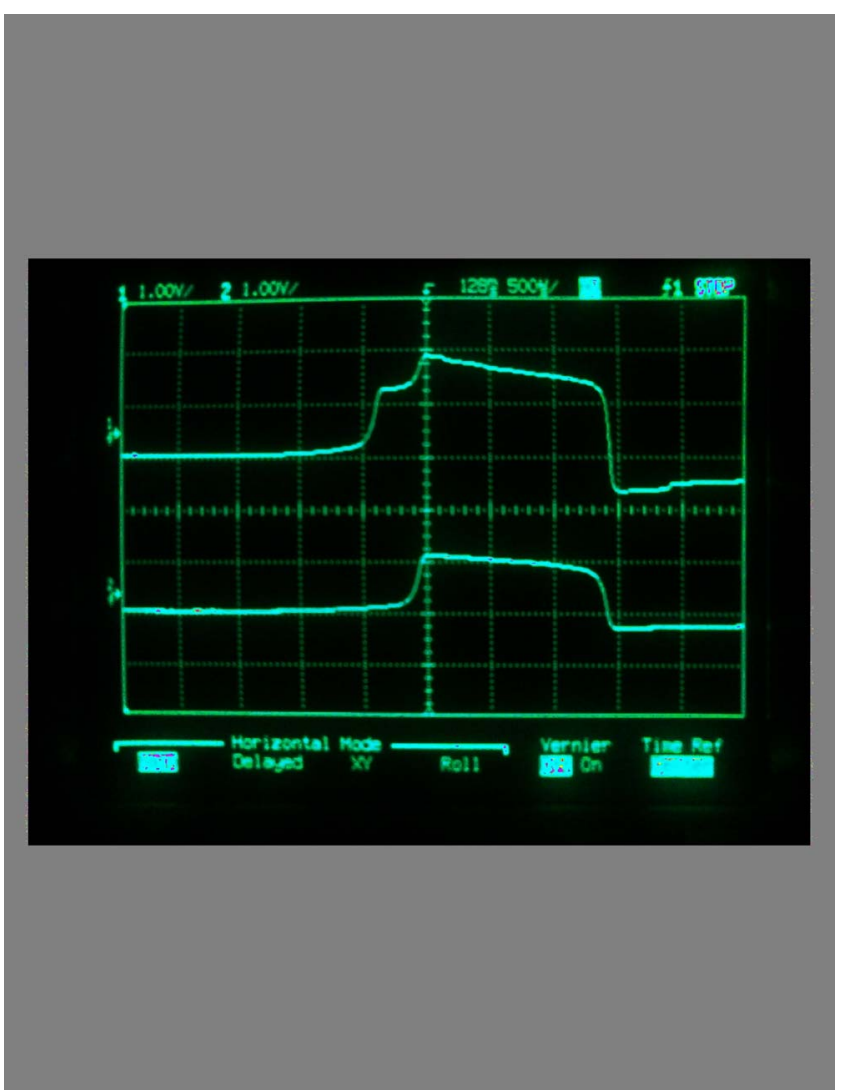

FIG. 5. (Color online) Trajectories of the master (upper trace) and slave systems (lower trace) in an experimental implementation of the anticipated synchronization scheme in an electronic circuit simulating the FitzHugh-Nagumo neuronal dynamics.

event, even for a zero response time, $t_{R}=0$, the condition $\epsilon_{0}>x_{0}-x_{+}$must be satisfied in order to bring back $x(t)$ from the threshold value $x_{0}$ to the stable region.

Our method works similarly but the decision of activating the corrective signal depends on the dynamics of the auxiliary system $y(t)$, coupled to $x(t)$ using the anticipated synchronization scheme discussed previously. The main idea is that the dynamics of $y(t)$ anticipates that of $x(t)$ and hence the correction criterion $y(t)>x_{0}$ happens earlier. Consequently, this is an important advantage of the method: a much smaller magnitude $\epsilon_{0}$ is needed to suppress the undesired firing. Alternatively, a larger reaction time can be used to decide whether to apply the correcting signal. The correction criterion is now modified to the following: if $y(t)$ crosses the threshold value $x_{0}$ then a deltalike correction of magnitude $-\epsilon_{0}$ is applied to the variable $x(t)$. The same conditions for reaction and recovery times discussed before also apply here.

We now compare in detail the control methods using the criterions based on the $y(t)$ or the $x(t)$ variables. When using, for example, a threshold value $x_{0}=\pi / 4$, our numerical results show that for a response time $t_{R}=0$, a value $\epsilon_{0}=0.468$ is needed when using the criterion on the $x(t)$ variable, while a much smaller value $\epsilon_{0}=0.022$ suffices in our scheme using the criterion based on $y(t)$. The same result applies in the case of a nonzero response time. If $t_{R}=1, \epsilon_{0}$ reduces from 0.834 , when using the criterion of $x(t)$, to 0.0307 , when using our method, or from 1.751 to 0.0426 if $t_{R}=2$. For $t_{R}=3$ the 


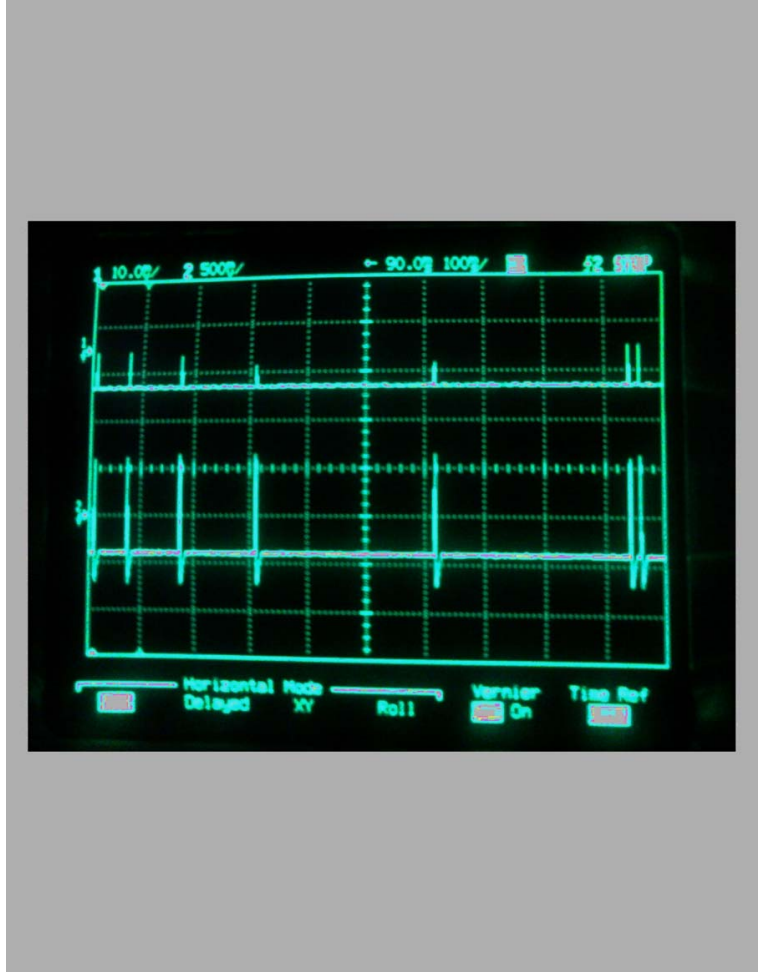

(a)

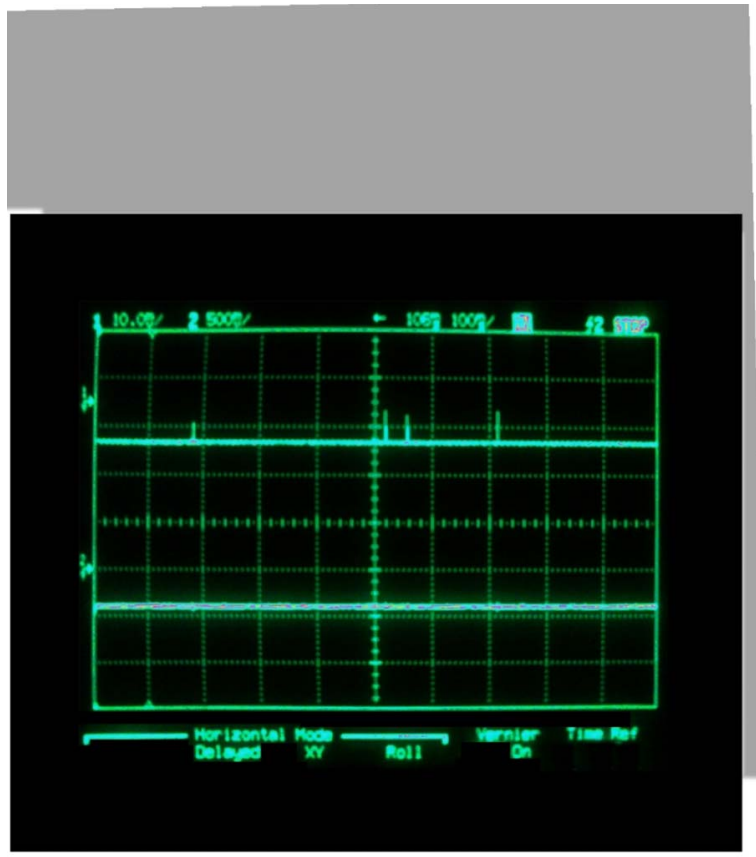

(b)

FIG. 6. (Color online) Experimental verification of the predict-control method in the electronic FitzHugh-Nagumo system. The small vertical lines at the top of each figure are the external perturbations, and the lower traces are the master's variable. In the right panel, the spikes have been controlled using a criterion based on the dynamics of the slave (not shown). The left panel shows that the same procedure fails to prevent the spikes if the criterion is applied using the master's variable.

method based on $x(t)$ is unable to control the system while for our method it suffices to take $\epsilon_{0}=0.0597$. In Fig. 2 we show that our control method is efficient and indeed enables the suppression of a single spike.

\section{B. Systems with noise}

In a typical control situation, the arrival of the perturbation and the consequent generation of spikes would be random. To model this, we consider a train of perturbations arriving at times $t_{i}$ such that the time intervals $t_{i+1}-t_{i}$ are distributed according to an exponential distribution of mean value $\langle t\rangle$. We also add to both Eqs. (3) and (4) independent white noise terms $D \xi(t)$ with correlations $\left\langle\xi(t) \xi\left(t^{\prime}\right)\right\rangle=\delta\left(t-t^{\prime}\right)$. The left panel of Fig. 3 shows the result of our control method while the right panel shows the control method based on the $x(t)$ variable. It can be observed that the intensity $\epsilon_{0}$ of the control is smaller with our method and the spikes are better suppressed. Finally, we have checked that our control method still works well when the auxiliary system is not an identical copy of the master system.

\section{Determination of the control region}

The control is efficient as long as the anticipation time between the master and slave is nonzero and positive. Only in such a case an ahead prevention is possible. Consequently it is important to estimate the parameter ranges for $\tau$ and $K$ for which the efficient control is possible. The results of Fig. 4 show that the anticipation times become smaller for stronger external perturbations in $x(t)$. This is due to the fact that when the perturbation is stronger the response time of $x(t)$ is faster. This induces a decreasing in the anticipation times since the highest possible anticipation times are bounded by the response times of the master (see Ref. [19] for details). In consequence the slow response systems or those that are slightly perturbed can be better and more efficiently controlled.

\section{EXPERIMENTAL IMPLEMENTATION}

In order to assess the practical usage of our method we have implemented experimentally an electronic version of the FitzHugh-Nagumo neurons, another type of excitable system [25]. The details concerning the experimental setup can be found in [16]. For this work the setup was modified introducing a monostable circuit that generates a pulse with programmable variable width and delay when the slave system signal crosses a threshold. The pulse is applied as a corrective signal to prevent firings of the master. The complete diagram of the setup can be found in [26]. Our experimental findings qualitatively agree with the numerical predictions. Figure 5 shows a snap shot taken from the oscilloscope showing the dynamics of the master and the slave systems with response to a perturbation applied only to the master. It can be clearly seen that the slave anticipates the 
firing of the master by roughly $400 \mu s$. In Fig. 6 we show time traces taken from the oscilloscope. The left panel shows the results when the master system is controlled using its own output. In this situation it is almost impossible to cancel the firing. The right panel shows the results using our control method based on the anticipating slave. Note that all spikes have been suppressed in this particular realization of the experiment, a typical situation.

\section{CONCLUSIONS}

In conclusion, we have proposed a predict-prevent control method and shown its feasibility for the suppression of spikes in perturbed excitable systems. The method consists of two steps: (1) prediction made by the slave system with the use of the anticipated synchronization scheme and (2) correction applied to the master in order to suppress its unwanted response. We have demonstrated numerically and experimentally the efficiency of the method.

Our method displays some of the most advantageous characteristics of the delayed-feedback control methods of Ott et al. [3] and Pyragas [4] but nevertheless differs essentially from them. The method uses the delayed feedback, as in the delayed-feedback control method, but the use of it has completely different scope: it is used in an auxiliary slave system to process the information (predict) and decide whether to activate or not the control. Once the decision of activating the control has been taken, a small perturbation is applied to the master in order to prevent its firing.

At variance with previous methods, our technique is more efficient because the real-time prediction by the slave allows for a fast decision making. It is also less invasive since it uses localized small feedback stimuli. The proposed technique has been applied to excitable systems but we hope that this work will stimulate additional studies in order to extend this method to other cases, such as cardiac and neural tissues, electric and electronic circuits, laser systems, etc., as well as to identify possible situations of practical interest.

\section{ACKNOWLEDGMENTS}

We acknowledge financial support by the MEC (Spain) and FEDER (EU) through Project No. FIS2007-60327, the EU NoE BioSim under Contract No. LSHB-CT-2004005137, and a Marie Curie action within the Sixth European Community Framework Programme.
[1] Katsuhiko Ogata, Modern Control Engineering (Prentice-Hall, Englewood Cliffs, NJ, 2001).

[2] A. V. Panfilov, Chaos 8, 57 (1998).

[3] E. Ott, C. Grebogi, and J. A. Yorke, Phys. Rev. Lett. 64, 1196 (1990).

[4] K. Pyragas, Phys. Lett. A 170, 421 (1992).

[5] J. E. S. Socolar, D. W. Sukow, and D. J. Gauthier, Phys. Rev. E 50, 3245 (1994).

[6] O. Lüthje, S. Wolff, and G. Pfister, Phys. Rev. Lett. 86, 1745 (2001).

[7] K. Hall, D. J. Christini, M. Tremblay, J. J. Collins, L. Glass, and J. Billette, Phys. Rev. Lett. 78, 4518 (1997).

[8] S. Sinha and W. L. Ditto, Phys. Rev. E 63, 056209 (2001).

[9] A. Pumir, V. Nikolski, M. Horning, A. Isomura, K. Agladze, K. Yoshikawa, R. Gilmour, E. Bodenschatz, and V. Krinsky, Phys. Rev. Lett. 99, 208101 (2007).

[10] A. Pikovsky, M. Rosenblum, and J. Kurths, Synchronization: A Universal Concept in Nonlinear Sciences (Cambridge University Press, New York, 2001).

[11] I. I. Blekhman, Synchronization: In Science and Technology (ASME, New York, 1988).

[12] H. U. Voss, Phys. Rev. E 61, 5115 (2000); 64, 039904(E) (2001); Phys. Rev. Lett. 87, 014102 (2001).

[13] O. Calvo, D. R. Chialvo, V. M. Eguiluz, C. Mirasso, and R. Toral, Chaos 14, 7 (2004).
[14] C. Masoller, Phys. Rev. Lett. 86, 2782 (2001).

[15] E. Hernández-García, C. Masoller, and C. R. Mirasso, Phys. Lett. A 295, 39 (2002).

[16] M. Ciszak, O. Calvo, C. Masoller, C. R. Mirasso, and R. Toral, Phys. Rev. Lett. 90, 204102 (2003).

[17] R. Toral, C. Masoller, C. Mirasso, M. Ciszak, and O. Calvo, Physica A 325, 192 (2003).

[18] M. Kostur, P. Hanggi, P. Talkner, and J. L. Mateos, Phys. Rev. E 72, 036210 (2005).

[19] M. Ciszak, F. Marino, R. Toral, and S. Balle, Phys. Rev. Lett. 93, 114102 (2004).

[20] Y. Liu, Y. Takiguchi, P. Davis, T. Aida, S. Saito, and J. M. Liu, Appl. Phys. Lett. 80, 4306 (2002).

[21] H. U. Voss, Int. J. Bifurcation Chaos Appl. Sci. Eng. 12, 1619 (2002).

[22] A. N. Pisarchik, R. Jaimes-Reategui, and J. H. Garcia-Lopez, Philos. Trans. R. Soc. London, Ser. A 366, 459 (2008).

[23] M. Ciszak, R. Toral, and C. Mirasso, Mod. Phys. Lett. B 18, 1135 (2004).

[24] R. Adler, Proc. IRE 34, 351 (1946); Proc. IEEE 61, 1380 (1973).

[25] R. FitzHugh, Biophys. J. 1, 445 (1961); J. Nagumo, S. Arimoto, and S. Yoshizawa, Proc. IRE 50, 2061 (1962).

[26] http://ifisc.uib-csic.es/claudio/activities.html 